Short contribution

\title{
Nutrient effects on cellulase production by the new species, Clostridium thermocopriae
}

Fengxie Jin* and Kiyoshi Toda

Institute of Applied Microbiology, University of Tokyo, Bunkyo-ku, Tokyo 113, Japan

In: Appl Microbiol Biotechnol 31:597-600

The above paper was intended to be a Short contribution. 